\title{
Density-Temperature Formulae for Coexisting Liquid and Vapor and for Freezing Liquid Parahydrogen
}

\author{
Robert D. Goodwin*
}

\author{
Institute for Materials Research, National Bureau of Standards, Boulder, Colo.
}

(June 21, 1966)

\begin{abstract}
A single formula is given for coexistence temperature as a continuous function of the vapor-liquid densities. With six coefficients and seven adjusted exponents, it may conveniently replace the several formulae formerly used in separate regions of the data. Freezing liquid densities are described by a simple power law in temperature, replacing more complicated formulae. Computed results are tabulated and compared with the derived data.
\end{abstract}

Key Words: Coexistence densities, freezing liquid densities, hydrogen, melting-line liquid densities, orthobaric densities, parahydrogen, vapor-liquid equilibrium.

\section{Introduction}

Data for densities of the coexisting phases of parahydrogen are derived, not directly experimental. They were obtained as intersections of $P V T$ isochores and isotherms with vapor-pressure and melting-line formulae for $P(T)$ at coexistence $[1,2] .{ }^{1}$ Any concise decription of these densities is a useful computational aid, as for example, to obtain heats of transition via the Clapeyron and $P(T)$ equations. In [1], however, we had used three formulae, in three regions of the orthobaric densities, with a total of 14 coefficients and about 12 adjusted exponents. The present search for a single formula for orthobaric densities was motivated by the successful application of Ehrlich's simple formula for saturated liquid [5] to our parahydrogen data. This is reported below.

Background on fluid behavior is given by [3], and quantum effects are under study [4]. Additional references on parahydrogen are given in the monograph [6]. Useful with the Clapeyron equation are recent determinations of heats of fusion [7] and of densities of solid parahydrogen [8]. The words: coexisting; at coexistence; orthobaric; at saturation; and saturated vapor, liquid or solid, all refer to one or more of at least two phases in equilibrium. The NBS-1955 low temperature scale used here is the same as in [6], and we define one liter, $\mathrm{L}$, to be $1000 \mathrm{~cm}^{3}$.

\section{Ehrlich's Formula for Saturated Liquid Parahydrogen}

Ehrlich's formula, eq (1), employs reduced variables, $\rho \equiv D / D_{c}, \tau \equiv T / T_{c}$ (where $D$ and $T$ are density and

\footnotetext{
* Cryogenics Division, NBS Laboratories, Boulder, Colo.

${ }^{1}$ Figures in brackets indicate the literature references at the end of this paper.
}

temperature, with subscript $c$ referring to the critical point),

$$
(\rho-1)^{3} / \rho=A \cdot(1-\tau)+B \cdot(1-\tau)^{2} .
$$

We used iterative methods and a digital computer to find the critical constants for eq (1) which minimize the mean relative deviation between calculated densities and 46 pairs of data for $\rho(\tau)$ [1]. This form of deviation was selected because the data are not directly experimental. Largest relative deviations occur near the critical point and are tabulated in summarizing table 1 , while those near the triple point are plotted in figure 1 as open circles. [We define $\Delta$, $\left.\% \equiv 100 \cdot\left(D_{\text {calc }}-D_{\text {ref }}\right) / D_{\text {ref. }}\right]$ Most of the remaining deviations are a mere few hundredths of 1 percent. It is seen that this formula gives an excellent representation of the data.

TABLE 1. Ehrlich's formula for saturated liquid parahydrogen

\begin{tabular}{|c|c|c|c|}
\hline $\begin{aligned} & \text { Constants } T_{\mathrm{c}} \\
& A\end{aligned}$ & $\begin{array}{l}2.98438 \\
85912\end{array}$ & $\begin{array}{l}D_{c}= \\
=-0 .\end{array}$ & $\begin{array}{l}7685 \mathrm{~g} \mathrm{~mol} \\
205\end{array}$ \\
\hline Mean deviati & 46 point & $=0.043$ & ent \\
\hline Maximum & ve deviat & is near & al point \\
\hline $\mathrm{T},{ }^{\circ} \mathrm{K}$ & $\Delta, \%$ & $\mathrm{~T},{ }^{\circ} \mathrm{K}$ & $\Delta, \%$ \\
\hline 32.914 & 0.000 & 32.363 & -0.044 \\
\hline 32.739 & -0.195 & 32.039 & -0.140 \\
\hline 32.579 & -0.110 & 32.000 & +0.038 \\
\hline
\end{tabular}

\section{A Formula for Densities of Coexisting Vapor and Liquid}

In attempting to modify Ehrlich's formula for densities below critical we note that as $\rho \rightarrow 0$ the familiar vapor-pressure and virial equations provide a relation between temperature and density,

$$
P \sim P_{0} \cdot \exp \left(-T_{0} / T\right) \sim D \cdot R \cdot T,
$$




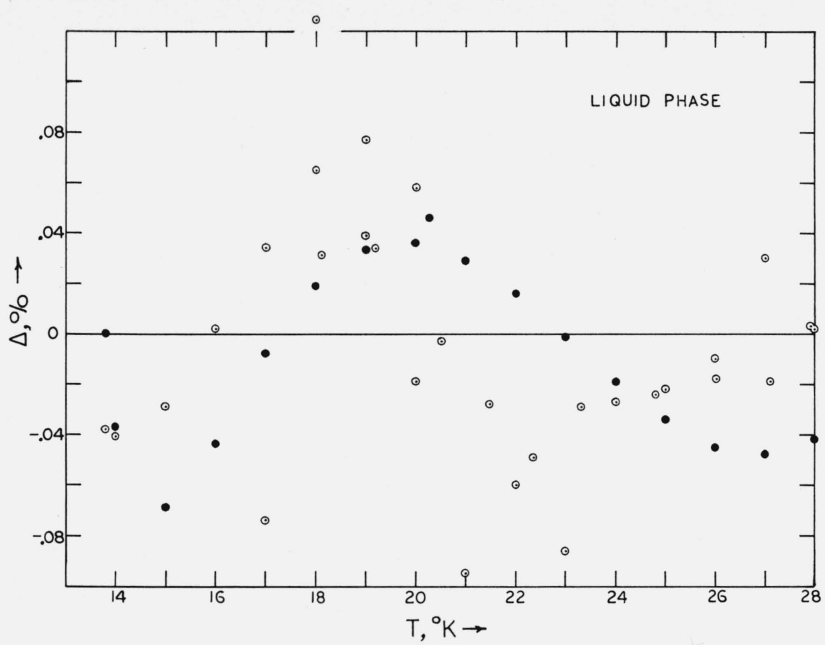

FIGURE 1. Comparison of calculated densities.

$\Delta \equiv 100 \cdot\left(D_{\text {calc }}-D_{\text {ret }}\right) / D_{\text {ref }}$ : Open circles, Ehrlich's formula with constants of table 1 , using for $D_{\text {ret }}$ the derived data of [1]; filled circles from table 4 , this report.

where $P$ is pressure, $R$ is the gas constant, and $P_{0}, T_{0}$ are constants. At coexistence, $D \cdot R \cdot T$ may be regarded as a function only of the density. Normalizing the above exponential at the critical point in the form, $\exp [\alpha \cdot(1-1 / \tau)]$ where $\alpha$ is a constant, and comparing its Taylor's series expansion about $\tau=1$ with eq (1), we have been led to the formula,

$$
\exp [\alpha \cdot(1-1 / \tau)]=1-(\rho-1)^{\beta / 3} / \exp [\rho \cdot f(\rho)],
$$

wherein $\beta / 3$ replaces exponent 3 from eq $(1)$, and $f(\rho)$ is discussed below. This is a single-valued expression for coexistence temperature as a function of the densities, constrained to a given critical point. As the variable $\tau$ ranges from 0 to +1 only, $\beta$ must be an even integer. The expression $\left[1-(\rho-1)^{\beta / 3}\right]$ becomes negative for $2<\rho \leqslant 2.45$ where $\rho=2.45$ is the upper limit for liquid parahydrogen at the triple point.

The exponential on the right side of eq (2) has been introduced as an empirical method for seeking representation of data. We let $f(\rho)$ be a polynomial, and search for the requisite number of terms and powers of $\rho$, finding coefficients by least-squares in the form $Y / \rho=f(\rho)$, where

$$
Y(\alpha, \beta, \rho, \tau) \equiv \log _{e}\left\{(\rho-1)^{\beta / 3} /\left[1-e^{\alpha(1-1 / \tau)}\right]\right\} .
$$

For each trial $\beta$ and each form, $f(\rho)$, the value of $\alpha$ is found by an iterative procedure with the high-speed computer. By requisite number of terms and powers of $\rho$ in this report we mean that combination which yields deviations comparable with uncertainty of the data, and a mean relative deviation less than 0.1 percent. We have used only the critical-point constants established by [1], not to be confused with the optimizing values in table 1.

With 75 pairs of data for $\tau(\rho)[1]$, the behavior of $Y$ versus $\rho$ is highly sensitive to the values of $\alpha$ and of $\beta$. For $\beta=6$ and for $\beta=10$ we find that $Y$ apparently

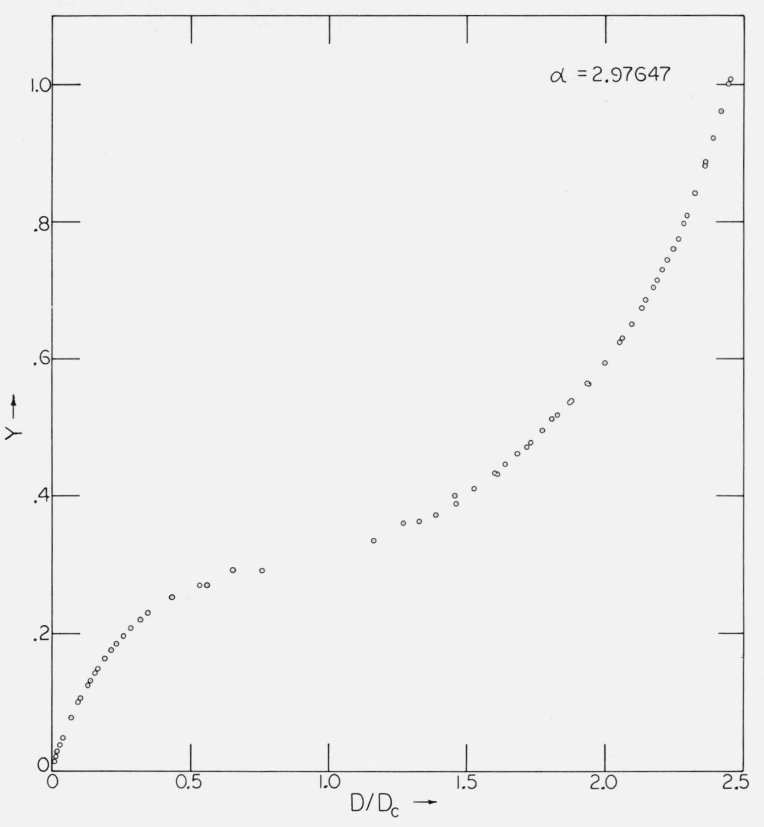

FiguRE 2. $\mathrm{Y}(\alpha, \beta, \rho, \tau)$ versus $\rho$ for $\alpha=2.97647, \beta=8$.

tends to infinite discontinuities as $\rho \rightarrow 1$, from below or from above, whereas for $\beta=8$ the behavior is seen in figure 2, for which $\alpha=2.97647, \beta=8$. This plot has the qualitative behavior of an isotherm of pressure versus density at temperatures a little above critical, suggesting a cubic polynomial in the density, [or a quadratic for $f(\rho)$ in eq (2)]. Tedious exploration of forms for $f(\rho)$, however, yields

$$
\begin{aligned}
f(\rho) \equiv A_{1}+A_{2} \cdot \rho^{-1 / 3} & +A_{3} \cdot \rho^{1 / 3} \\
& +A_{4} \cdot \rho^{8 / 3}+A_{5} \cdot \rho^{3}+A_{6} \cdot \rho^{7},
\end{aligned}
$$

to complete eq (2) with above values for $\alpha, \beta$. Integer exponents only were explored for the last two terms in eq (3). Mean relative deviation of temperatures using eq (3) is 0.0624 percent. As compared with a three-term expression for $f(\rho)$, we note that an additional term, $A_{2} \cdot \rho^{-1 / 3}$, has been required for data at the lowest densities, and an opposition of similar terms, $A_{4} \cdot \rho^{8 / 3}+A_{5} \cdot \rho^{9 / 3}$, (with opposite signs) apparently is required for the critical region (fig. 2).

TABLE 2. Constants for eqs (2), (3), parahydrogen

\begin{tabular}{c|l}
\hline \hline & \\
$D_{c}=15.59 \mathrm{~g} \mathrm{~mol} / \mathrm{L}$, & $T_{c}=32.976{ }^{\circ} \mathrm{K}$ \\
$\alpha=2.97647$, & $\beta=8$, \\
$A_{1}=+2.0300583$, & $A_{2}=-0.0587951$, \\
$A_{3}=-1.8565706$, & $A_{4}=+0.6205095$, \\
$A_{5}=-0.42181139$, & $A_{6}=+0.6825745 \cdot 10^{-3}$ \\
\hline
\end{tabular}

Table 2 gives constants for eqs (2) and (3). Table 3 compares calculated temperatures from eq (2) with the data. Table 4 gives densities obtained from eq (2) by an iterative method at integral temperatures, and compares them with densities calculated in [1] with 


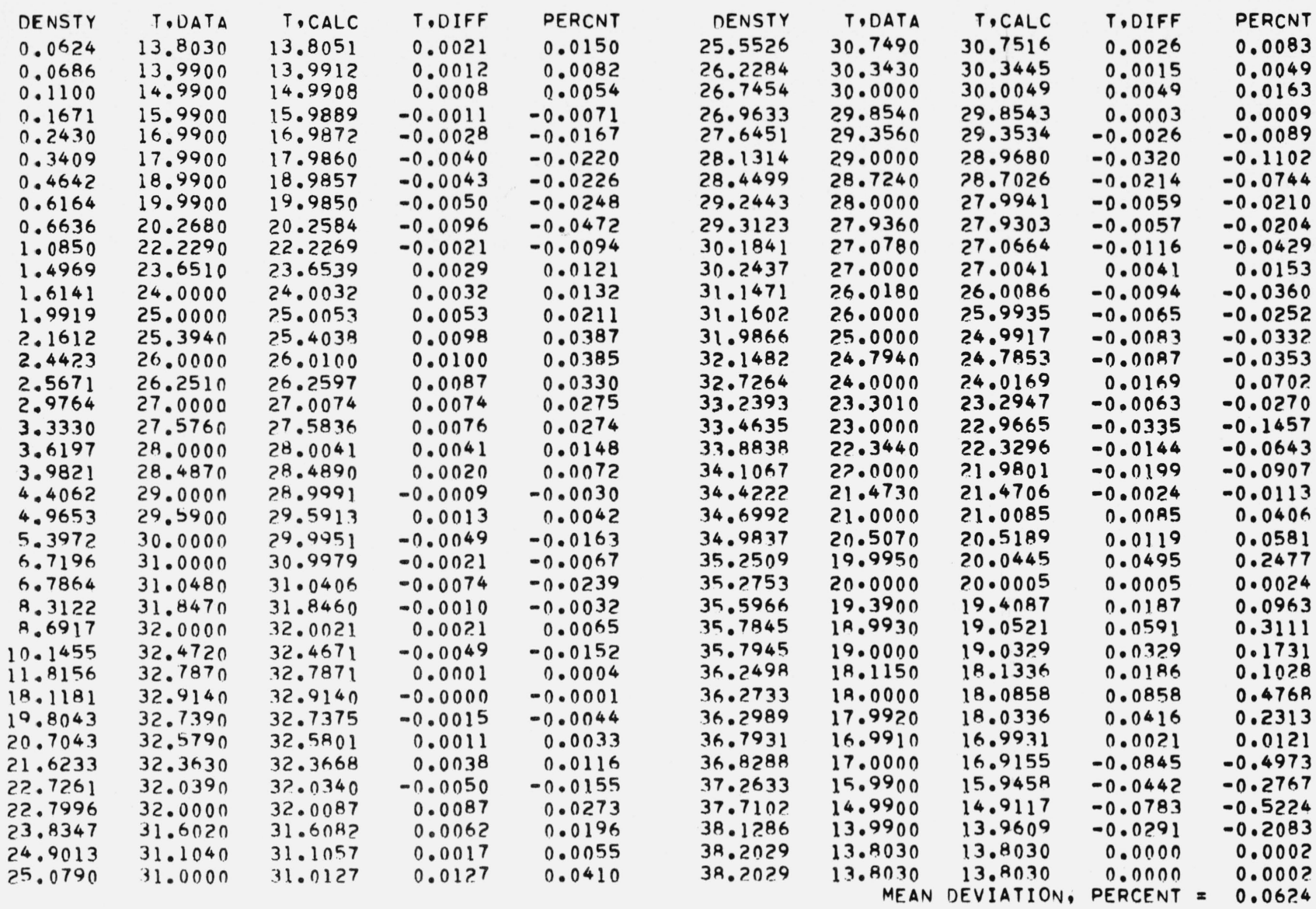

the more elaborate formulae. Table 5 gives temperatures from eq (2) at uniformly spaced densities and compares them with values from the monograph [6].

Deviations of temperature (table 3) are relatively large and uniform for liquid densities approaching the triple point, giving alarm that the large exponents used in eq (3) may be responsible. From [6] we find, however, that $(\rho / \tau)(d \tau / d \rho) \approx 3$ to 6 in this region, so that relative temperature deviations will be roughly fivefold greater than corresponding density deviations. We note, also, the experimentalist's estimate of 0.1 percent accuracy [9] for much of the density data used in [1]. Turning to deviations in density, we see in figure 1 that those from eq (2), filled circles, are smaller than or comparable with those from the simple formula eq (1). [To avoid a multiplicity of tables, we have compared densities from eq (2) with the sufficiently precise, calculated results from [1]; hence the relative difference also is a smooth function.] It appears highly improbable that deviations of eq (2) are due entirely to the form of $f(\rho)$ in eq (3). With data for other substances, however, we may expect that this form should be modified.
TABLE 4. Comparison of calculated densities from eq (2) at uniform temperatures with calculated data of [1]

CALCULATED DENSITIES, GMOL/L VAPOR PHASE LIQUID PHASE

T.DG.K CALCLTD REFRNCE DIFRNCE CALCLTD REFRNCE DIFRNCE $13.803 \quad 0.0623 \quad 0.0624-0.000138 .203038 .2068=0.0038$ $14.000 \quad 0.0689 \quad 0.0690-0.000138 .1105 \quad 38.1246 \quad-0.0141$ $15.000 \quad 0.1104 \quad 0.1104 \quad 0.0000 \quad 37.6723 \quad 37.6984=0.0261$ $16.000 \quad 0.1678 \quad 0.1678 \quad 0.0000 \quad 37.2395 \quad 37.2552 \quad-0.0157$ $17.000 \quad 0.2441 \quad 0.2440 \quad 0.000136 .789936 .7928=0.0029$

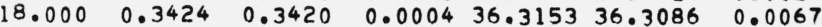
$\begin{array}{llllllll}19.000 & 0.4662 & 0.4653 & 0.0009 & 35.8116 & 35.7997 & 0.0119\end{array}$ $\begin{array}{lllllll}20.000 & 0.6189 & 0.6176 & 0.0013 & 35.2755 & 35.2628 & 0.0127\end{array}$ $\begin{array}{lllllll}20.268 & 0.6653 & 0.6636 & 0.0017 & 35.1260 & 35.1100 & 0.0160\end{array}$ $\begin{array}{lllllll}21.000 & 0.8048 & 0.8030 & 0.0018 & 34.7042 & 34.6940 & 0.0102\end{array}$ $\begin{array}{llllllll}22.000 & 1.0284 & 1.0273 & 0.0011 & 34.0941 & 34.0886 & 0.0055\end{array}$ $23.000 \quad 1.2955 \quad 1.2956-0.000133 .440933 .4411-0.0002$ $24.000 \quad 1.6130 \quad 1.6145 \quad-0.0015 \quad 32.7387 \quad 32.7448 \quad-0.0061$ $25.000 \quad 1.9897 \quad 1.9926-0.002931 .980131 .9910-0.0109$ $26.000 \quad 2.4374 \quad 2.4413-0.0039 \quad 31.1546 \quad 31.1685-0.0139$ $\begin{array}{lllllllll}27.000 & 2.9721 & 2.9762 & -0.0041 & 30.2476 & 30.2622 & -0.0146\end{array}$ $\begin{array}{llllllll}28.000 & 3.6168 & 3.6201 & -0.0033 & 29.2380 & 29.2502 & -0.0122\end{array}$ $29.000 \quad 4.4070 \quad 4.4082-0.0012 \quad 28.0922 \quad 28.0991-0.0069$ $\begin{array}{lllllll}30.000 & 5.4027 & 5.4006 & 0.0021 & 26.7526 & 26.7520 & 0.0006\end{array}$ $\begin{array}{llllllll}31.000 & 6.7228 & 6.7170 & 0.0058 & 25.1029 & 25.0946 & 0.0083\end{array}$ $\begin{array}{lllllll}32.000 & 8.6864 & 8.6800 & 0.0064 & 22.8246 & 22.8145 & 0.0101\end{array}$ $\begin{array}{llllllll}32.400 & 9.8926 & 9.8880 & 0.0046 & 21.4947 & 21.4887 & 0.0060\end{array}$ $32.700 \quad 11.2457 \quad 11.2425 \quad 0.0032 \quad 20.045920 .0463 \quad-0.0004$ $32.90012 .899712 .8921 \quad 0.007618 .320318 .3247-0.0044$ 
TABLE 5. Comparison of calculated temperatures from eq (2) at uniform densities with derived results from [6]

\begin{tabular}{|c|c|c|c|c|c|c|c|}
\hline \multirow{2}{*}{ Density } & \multicolumn{3}{|c|}{ Temperature, deg. $\mathrm{K}$} & \multirow{2}{*}{ Density } & \multicolumn{3}{|c|}{ Temperature, deg. K } \\
\hline & Calculated & Reference & Difference & & Calculated & Reference & Difference \\
\hline 0.5 & 19.240 & 19.243 & -0.003 & 19.5 & 32.780 & 32.780 & 0.000 \\
\hline 1.0 & 21.882 & 21.887 & -0.005 & 20.0 & 32.707 & 32.707 & 0.000 \\
\hline 1.5 & 23.663 & 23.660 & 0.003 & 20.5 & 32.620 & 32.620 & 0.000 \\
\hline 2.0 & 25.025 & 25.018 & 0.007 & 21.0 & 32.517 & 32.517 & 0.000 \\
\hline 2.5 & 26.127 & 26.119 & 0.008 & 21.5 & 32.399 & 32.397 & 0.002 \\
\hline 3.0 & 27.048 & 27.041 & 0.007 & 22.0 & 32.263 & 32.261 & 0.002 \\
\hline 3.5 & 27.833 & 27.828 & 0.005 & 22.5 & 32.109 & 32.106 & 0.003 \\
\hline 4.0 & 28.512 & 28.509 & 0.003 & 23.0 & 31.938 & 31.934 & 0.004 \\
\hline 4.5 & 29.104 & 29.103 & 0.001 & 23.5 & 31.747 & 31.742 & 0.005 \\
\hline 5.0 & 29.625 & 29.626 & -0.001 & 24.0 & 31.536 & 31.532 & 0.004 \\
\hline 5.5 & 30.085 & 30.087 & -0.002 & 24.5 & 31.306 & 31.301 & 0.005 \\
\hline 6.0 & 30.492 & 30.495 & -0.003 & 25.0 & 31.054 & 31.050 & 0.004 \\
\hline 6.5 & 30.853 & 30.856 & -0.003 & 25.5 & 30.782 & 30.778 & 0.004 \\
\hline 7.0 & 31.172 & 31.176 & -0.004 & 26.0 & 30.487 & 30.484 & 0.003 \\
\hline 7.5 & 31.455 & 31.459 & -0.004 & 26.5 & 30.169 & 30.168 & 0.001 \\
\hline 8.0 & 31.705 & 31.709 & -0.004 & 27.0 & 29.828 & 29.829 & -0.001 \\
\hline 8.5 & 31.925 & 31.928 & -0.003 & 27.5 & 29.464 & 29.466 & -0.002 \\
\hline 9.0 & 32.118 & 32.120 & -0.002 & 28.0 & 29.075 & 29.080 & -0.005 \\
\hline 9.5 & 32.285 & 32.287 & -0.002 & 28.5 & 28.660 & 28.668 & -0.008 \\
\hline 10.0 & 32.429 & 32.430 & -0.001 & 29.0 & 28.219 & 28.229 & -0.010 \\
\hline 10.5 & 32.552 & 32.553 & -0.001 & 29.5 & 27.752 & 27.764 & -0.012 \\
\hline 11.0 & 32.656 & 32.656 & -0.000 & 30.0 & 27.256 & 27.270 & -0.014 \\
\hline 11.5 & 32.741 & 32.742 & -0.001 & 30.5 & 26.732 & 26.747 & -0.015 \\
\hline 12.0 & 32.811 & 32.811 & -0.000 & 31.0 & 26.178 & 26.194 & -0.016 \\
\hline 12.5 & 32.866 & 32.866 & -0.000 & 31.5 & 25.592 & 25.608 & -0.016 \\
\hline 13.0 & 32.907 & 32.908 & -0.001 & 32.0 & 24.975 & 24.988 & -0.013 \\
\hline 13.5 & 32.937 & 32.938 & -0.001 & 32.5 & 24.323 & 24.334 & -0.011 \\
\hline 14.0 & 32.957 & 32.958 & -0.001 & 33.0 & 23.637 & 23.642 & -0.005 \\
\hline 14.5 & 32.969 & 32.970 & -0.001 & 33.5 & 22.912 & 22.912 & 0.000 \\
\hline 15.0 & 32.975 & 32.975 & -0.000 & 34.0 & 22.148 & 22.141 & 0.007 \\
\hline 15.5 & 32.976 & 32.976 & -0.000 & 34.5 & 21.342 & 21.327 & 0.015 \\
\hline 16.0 & 32.976 & 32.975 & 0.001 & 35.0 & 20.490 & 20.469 & 0.021 \\
\hline 16.5 & 32.972 & 32.972 & -0.000 & 35.5 & 19.589 & 19.564 & 0.025 \\
\hline 17.0 & 32.963 & 32.963 & -0.000 & 36.0 & 18,633 & 18.612 & 0.021 \\
\hline 17.5 & 32.946 & 32.947 & -0.001 & 36.5 & 17.618 & 17.609 & 0.009 \\
\hline 18.0 & 32.921 & 32.922 & -0.001 & 37.0 & 16.539 & 16.556 & -0.017 \\
\hline 18.5 & 32.886 & 32.886 & 0.000 & 37.5 & 15.401 & 15.451 & -0.050 \\
\hline 19.0 & 32.839 & 32.840 & -0.001 & 38.0 & 14.245 & 14.295 & -0.050 \\
\hline
\end{tabular}

\section{A Formula for Densities of Freezing Liquid}

In [2], densities of freezing liquid were derived as intersections of $P V T$ isochores and isotherms with an analytically described $P(T)$ melting line, and given a rather complicated, empirical description. We now find that a simple power law [eq (4)] is sufficient. Variables are normalized at the triple point (subscript $t$ ):

$$
\left(D / D_{t}\right)=\left(T / T_{t}\right)^{\gamma} .
$$

For parahydrogen, the constants used are $D_{t}=(1.0 /$ $0.026176) \mathrm{g} \mathrm{mol} / L, T_{t}=13.803^{\circ} \mathrm{K}[10]$, and we find the exponent, $\gamma=0.3104277$ by minimizing the mean relative deviation. Table 6 shows that eq (4) gives adequate representation. The first four columns are from [2].

The number of digits, given for constants in tables 1,2 , and for $\gamma$ in eq (4), is more than sufficient to reproduce the calculated results, and is not the result of statistical analysis.

Mr. William J. Hall contributed the essential computer programs for iteration and for least squares.
TABLE 6. Comparison of calculated densities of freezing liquid with derived data from [2] ${ }^{\mathrm{a}}$

\begin{tabular}{|c|c|c|c|c|c|}
\hline \multirow{2}{*}{ T, deg. K } & \multicolumn{3}{|c|}{ Publication } & \multicolumn{2}{|c|}{ Above formula } \\
\hline & D, data & $\mathrm{D}$, calc & D, percent & D, calc & $\mathrm{D}$, percent \\
\hline 14.171 & 38.50 & 38.51 & 0.03 & 38.516 & 0.042 \\
\hline 14.416 & 38.70 & 38.72 & 0.05 & 38.722 & 0.056 \\
\hline 14.764 & 39.00 & 39.00 & $0.0 \mathrm{I}$ & 39.010 & 0.024 \\
\hline 15.247 & 39.39 & 39.40 & 0.01 & 39.401 & 0.029 \\
\hline 15.375 & 39.50 & 39.50 & 0.01 & 39.504 & 0.009 \\
\hline 16.006 & 40.00 & 39.99 & -0.01 & 40.000 & 0.000 \\
\hline 16.030 & 40.02 & 40.01 & -0.02 & 40.019 & -0.003 \\
\hline 16.655 & 40.50 & 40.49 & -0.02 & 40.497 & -0.008 \\
\hline 16.790 & 40.60 & 40.59 & -0.01 & 40.598 & -0.004 \\
\hline 17.000 & 40.76 & 40.75 & -0.02 & 40.755 & -0.012 \\
\hline 17.323 & 41.00 & 40.99 & -0.02 & 40.994 & -0.015 \\
\hline 506 & 41.13 & 41. & -0.01 & 41.128 & -0.005 \\
\hline 000 & 41.49 & 41.49 & -0.00 & 41.485 & -0.013 \\
\hline 18.012 & 41.50 & 41.49 & -0.01 & 41.493 & -0.016 \\
\hline 18.214 & 41.65 & 41.64 & -0.02 & 41.637 & -0.031 \\
\hline 18.723 & 42.00 & 42.00 & -0.01 & 41.995 & -0.012 \\
\hline 883 & 42.11 & 42.11 & -0.01 & 42.106 & -0.009 \\
\hline 19.000 & 42.19 & 42.19 & -0.00 & 42.187 & -0.007 \\
\hline 19.455 & 42.50 & 42.50 & 0.00 & 42.498 & -0.005 \\
\hline 19.585 & 42.58 & 42.59 & 0.02 & 42.586 & 0.014 \\
\hline 20.000 & 42.86 & 42.87 & 0.02 & 42.864 & 0.009 \\
\hline 203 & 43.00 & 43.00 & 0.0 & 42.999 & -0.003 \\
\hline 218 & 43.01 & 43.01 & 0.01 & 43.009 & -0.003 \\
\hline 879 & 43.44 & 43.44 & 0.00 & 43.440 & 0.000 \\
\hline 20.976 & 43.50 & 43.50 & 0.01 & 43.503 & 0.006 \\
\hline 21.000 & 43.5 & 43.52 & 0.00 & 43.518 & -0.004 \\
\hline 21.468 & 43.81 & 43.81 & 0.01 & 43.817 & 0.016 \\
\hline 21.770 & 44.00 & 44.00 & 0.00 & 44.007 & 0.017 \\
\hline 22.000 & 44.14 & 44.14 & 0.01 & 44.151 & 0.025 \\
\hline 23.000 & 44.77 & 44.74 & -0.05 & 44.765 & -0.012 \\
\hline
\end{tabular}

a Densities in $\mathrm{g} \mathrm{mol} / \mathrm{L}$.

This work was supported by the National Aeronautics and Space Administration.

\section{References}

[1] H. M. Roder, D. E. Diller, L. A. Weber, and R. D. Goodwin, The orthobaric densities of parahydrogen, derived heats of vaporization, and critical constants, Cryogenics 3, No. 1, $16-22$ (1963).

[2] R. D. Goodwin and H. M. Roder, PVT relations of freezing liquid parahydrogen to 350 atm., Cryogenics 3, No. 1, 12-15 (1963).

[3] J. S. Rowlinson, Liquids and Liquid Mixtures, Butterworths, London, 1959.

[4] M. E. Fisher, Quantum corrections to critical point behavior, Phys. Rev. Letters 16, No. 1, 11-14 (1966).

[5] Percy Ehrlich, p. 49 in Advances in thermophysical properties Am. Soc. Mech. Engrs., New York, N.Y. 10017 (1965).

[6] H. M. Roder, L. A. Weber, and R. D. Goodwin, Thermodynamic and related properties of parahydrogen from the triple point to $100{ }^{\circ} \mathrm{K}$ at pressures to 340 atmospheres, NBS Monograph 94, August, 1965.

[7] R. F. Dwyer, G. A. Cook, B. M. Shields, and D. H. Stellrecht, Heat of fusion of solid parahydrogen, J. Chem. Phys. 42, No. 11, 3809-3812 (1965).

[8] R. F. Dwyer, G. A. Cook, O. E. Berwaldt, and H. E. Nevins, Molar volume of solid parahydrogen along the melting line, J. Chem. Phys. 43, No. 3, 801-805 (1965).

[9] R. D. Goodwin, Apparatus for determination of PVT relations and specific heats of hydrogen to $350 \mathrm{~atm}$ at temperatures above $14{ }^{\circ} \mathrm{K}$, J. Res. NBS 65C (Phys. and Chem.) No. 4, 231-243 (1961)

[10] H. W. Woolley, R. B. Scott, and F. G. Brickwedde, Compilation of thermal properties of hydrogen in its various isotopic and ortho-para modifications, J. Res. NBS 41, 379-475 (1948) RP1932.

(Paper 70A6-425) 